\title{
NuSTAR Reveals the Comptonizing Corona of the Broad-Line Radio Galaxy 3C 382
}

Ballantyne, D. R.; Bollenbacher, J. M.; Brenneman, L. W.; Madsen, K. K.; Balokovic, M.; Boggs, S. E.; Christensen, Finn Erland; Craig, W. W.; Gandhi, P.; Hailey, C. J.

Total number of authors:

17

Published in:

Astrophysical Journal

Link to article, DOI:

10.1088/0004-637X/794/1/62

Publication date:

2014

Document Version

Publisher's PDF, also known as Version of record

Link back to DTU Orbit

Citation (APA):

Ballantyne, D. R., Bollenbacher, J. M., Brenneman, L. W., Madsen, K. K., Balokovic, M., Boggs, S. E., Christensen, F. E., Craig, W. W., Gandhi, P., Hailey, C. J., Harrison, F. A., Lohfink, A. M., Marinucci, A.,

Markwardt, C. B., Stern, D., Walton, D. J., \& Zhang, W. W. (2014). NuSTAR Reveals the Comptonizing Corona of the Broad-Line Radio Galaxy 3C 382. Astrophysical Journal, 794(1). https://doi.org/10.1088/0004$637 X / 794 / 1 / 62$

\section{General rights}

Copyright and moral rights for the publications made accessible in the public portal are retained by the authors and/or other copyright owners and it is a condition of accessing publications that users recognise and abide by the legal requirements associated with these rights.

- Users may download and print one copy of any publication from the public portal for the purpose of private study or research.

- You may not further distribute the material or use it for any profit-making activity or commercial gain

- You may freely distribute the URL identifying the publication in the public portal 


\title{
NuSTAR REVEALS THE COMPTONIZING CORONA OF THE BROAD-LINE RADIO GALAXY 3C 382
}

\author{
D. R. Ballantyne ${ }^{1}$, J. M. Bollenbacher ${ }^{1}$, L. W. Brenneman ${ }^{2}$, K. K. Madsen $^{3}$, M. Baloković ${ }^{3}$, S. E. Boggs ${ }^{4}$,

 \\ C. B. MarkWARdt ${ }^{11}$, D. Stern ${ }^{12}$, D. J. WaLton ${ }^{3}$, AND W. W. Zhang ${ }^{11}$ \\ ${ }^{1}$ Center for Relativistic Astrophysics, School of Physics, Georgia Institute of Technology, \\ Atlanta, GA 30332, USA; david.ballantyne@physics.gatech.edu \\ ${ }^{2}$ Harvard-Smithsonian CfA, 60 Garden Street MS-67, Cambridge, MA 02138, USA \\ ${ }^{3}$ Cahill Center for Astronomy and Astrophysics, California Institute of Technology, Pasadena, CA 91125, USA \\ ${ }^{4}$ Space Science Laboratory, University of California, Berkeley, CA 94720 , USA \\ ${ }^{5}$ DTU SpaceNational Space Institute, Technical University of Denmark, Elektrovej 327, DK-2800 Lyngby, Denmark \\ ${ }^{6}$ Lawrence Livermore National Laboratory, Livermore, CA 94550, USA \\ ${ }^{7}$ Department of Physics, University of Durham, South Road, Durham DH1 3LE, UK \\ ${ }^{8}$ Columbia Astrophysics Laboratory, Columbia University, New York, NY 10027, USA \\ ${ }^{9}$ Department of Astronomy, University of Maryland, College Park, MD 20742-2421, USA \\ ${ }^{10}$ Dipartimento di Matematica e Fisica, Università degli Studi Roma Tre, via della Vasca Navale 84, I-00146 Roma, Italy \\ ${ }^{11}$ NASA Goddard Space Flight Center, Greenbelt, MD 20771, USA \\ 12 Jet Propulsion Laboratory, California Institute of Technology, Pasadena, CA 91109, USA \\ Received 2014 May 13; accepted 2014 August 14; published 2014 September 24
}

\begin{abstract}
Broad-line radio galaxies (BLRGs) are active galactic nuclei that produce powerful, large-scale radio jets, but appear as Seyfert 1 galaxies in their optical spectra. In the X-ray band, BLRGs also appear like Seyfert galaxies, but with flatter spectra and weaker reflection features. One explanation for these properties is that the X-ray continuum is diluted by emission from the jet. Here, we present two NuSTAR observations of the BLRG 3C 382 that show clear evidence that the continuum of this source is dominated by thermal Comptonization, as in Seyfert 1 galaxies. The two observations were separated by over a year and found 3C 382 in different states separated by a factor of 1.7 in flux. The lower flux spectrum has a photon-index of $\Gamma=1.68_{-0.02}^{+0.03}$, while the photon-index of the higher flux spectrum is $\Gamma=1.78_{-0.03}^{+0.02}$. Thermal and anisotropic Comptonization models provide an excellent fit to both spectra and show that the coronal plasma cooled from $k T_{e}=330 \pm 30 \mathrm{keV}$ in the low flux data to $231_{-88}^{+50} \mathrm{keV}$ in the high flux observation. This cooling behavior is typical of Comptonizing corona in Seyfert galaxies and is distinct from the variations observed in jet-dominated sources. In the high flux observation, simultaneous Swift data are leveraged to obtain a broadband spectral energy distribution and indicates that the corona intercepts $\sim 10 \%$ of the optical and ultraviolet emitting accretion disk. 3C 382 exhibits very weak reflection features, with no detectable relativistic $\mathrm{Fe}$ $\mathrm{K} \alpha$ line, that may be best explained by an outflowing corona combined with an ionized inner accretion disk.
\end{abstract}

Key words: accretion, accretion disks - galaxies: active - galaxies: individual (3C 382) - galaxies: nuclei X-rays: galaxies

Online-only material: color figures

\section{INTRODUCTION}

An important problem in extragalactic astrophysics is understanding the physical triggers that allow a small fraction of active galactic nuclei (AGNs) to produce powerful, large-scale radio jets. X-ray spectroscopic observations of AGNs are the most direct probe of the complex interactions of magnetic fields, fluid dynamics and relativistic physics that occur close to the central black hole (e.g., Reynolds \& Nowak 2003), and thus have the potential to make significant progress in elucidating the physical triggers of jetted AGNs (e.g., Ballantyne 2007; Lohfink et al. 2013). Observations of the brightest unobscured jetted AGNs, the broad-line radio galaxies (BLRGs), have consistently shown weaker reflection features and flatter X-ray spectra than typical Seyfert 1 galaxies (e.g., Sambruna et al. 1999; Eracleous et al. 2000; Grandi et al. 2001; Zdziarski \& Grandi 2001; Ballantyne 2007; Sambruna et al. 2009; Evans et al. 2010). In particular, the $\mathrm{Fe} \mathrm{K} \alpha$ lines are often observed to be narrow, low equivalent width (EW) features with a relativistic component from the inner accretion disk only rarely detectable (e.g., Lohfink et al. 2013). A number of explanations have been proposed to account for the weak reflection signatures in BLRGs including high inner disk ionization (Ballantyne et al. 2002), a change in the inner disk geometry (Eracleous et al. 2000; Lohfink et al. 2013), obscuration of the central accretion flow by the jet (Sambruna et al. 2009), black holes with retrograde spin (Garofalo et al. 2010; Evans et al. 2010), and dilution of the X-ray spectrum by jet emission (Grandi et al. 2002).

The broadband (3-79 keV), high sensitivity spectra provided by the focusing hard X-ray telescopes on the Nuclear Spectroscopic Telescope Array (NuSTAR; Harrison et al. 2013) observatory have the potential to be crucial in determining the correct interpretation of the BLRG spectra. The wide energy range allows for an accurate separation of the reflection and primary continua, as well as a more precise determination of the ionization state of the reflector (e.g., Risaliti et al. 2013; Brenneman et al. 2014a, 2014b; Marinucci et al. 2014). Moreover, if the $\mathrm{X}$-ray continuum of a BLRG is dominated by a Comptonizing corona (as in Seyfert 1 galaxies; e.g., Petrucci et al. 2000, 2001) then the high-energy sensitivity of NUSTAR will allow for measurements of the temperature $\left(k T_{e}\right)$ and optical depth $(\tau)$ of the plasma. In this way, NuSTAR data may finally start placing some 



Figure 1. Fullband (3-79 keV), background-subtracted NuSTAR lightcurves of 3C 382 during Observations 1 (left; $1000 \mathrm{~s}$ binning) and 2 (right; $3000 \mathrm{~s}$ binning). Data from FPMA and FPMB were summed to determine both lightcurves. The lightcurves are well described by a model with a constant count-rate: $\chi^{2} /$ dof $=24 / 22$ and $\chi^{2} /$ dof $=51 / 51$ for Observations 1 and 2, respectively. The low point in the light curve of Observation 1 only appears in the FPMA data, so does not indicate true variability from $3 \mathrm{C} 382$

Table 1

3C 382 Observation Log

\begin{tabular}{lccccc}
\hline \hline & Telescope & Observation ID & UT Start Date & Exposure (ks) & Counts \\
\hline Observation 1 & NuSTAR & 60061286002 & 2012 Sep 18 & $16.6 / 16.6$ & $20367 / 19988$ \\
(High flux) & Swift-XRT & 00080217001 & 2012 Sep 18 & 6.6 & 3678 \\
$\begin{array}{l}\text { Observation 2 } \\
\text { (Low flux) }\end{array}$ & NuSTAR & 60001084002 & 2013 Dec 18 & $82.6 / 82.4$ & $63596 / 61219$ \\
& & & & &
\end{tabular}

Notes. For the NUSTAR observations, exposure times and counts are listed for the two focal plane modules (FPMA/FPMB). The NuSTAR counts are the 3-79 keV background subtracted counts. Similarly, the background subtracted $0.3-7 \mathrm{keV}$ counts are indicated for the Swift-XRT data.

physical constraints on the interpretation of the X-ray spectra of BLRGs.

Here, we report on two $N U S T A R$ observations of the bright $\left(F_{2-10 \mathrm{keV}} \approx 3-6 \times 10^{-11} \mathrm{erg} \mathrm{cm}^{-2} \mathrm{~s}^{-1}\right.$; Gliozzi et al. 2007), nearby $(z=0.058)$ BLRG 3C 382. The source was detected in two different flux states that were separated by a factor of 1.7 , thus spanning the historical observed range. These observations allow detailed Comptonization modeling for different radiative conditions within the object. The next section describes the details of the NuSTAR observations, and the spectral analysis is presented in Section 3. Finally, Section 4 contains a discussion of the results.

\section{OBSERVATIONS}

Table 1 lists the details of the NuSTAR observations of 3C 382. Both NuSTAR data sets were reduced and spectral products extracted following standard procedures and using NuSTARDAS v.1.3.1 (included in HEASoft v.6.15.1) and NUSTAR CALDB 20131223. Source data from both focal plane modules (FPMA and FPMB) were extracted using a circular region of radius $60^{\prime \prime}$ centered on $3 \mathrm{C} 382$. A $80^{\prime \prime}$ radius source-free region on the same chip of the detector plane was used to extract the background in Observation 1, while a 95" radius region was used for the background region in Observation 2. The fullband, background-subtracted lightcurves of 3C 382 (summed from FPMA and FPMB) are shown in Figure 1. 3C 382 exhibited very little variability during both observations, consistent with earlier observations of this source (Grandi et al. 2001; Gliozzi et al. 2007; Sambruna et al. 2011). Fitting the light- curves (with $1000 \mathrm{~s}$ binning for Observation 1, and $3000 \mathrm{~s}$ binning for Observation 2) with a constant gives a $\chi^{2}$ per degree of freedom (dof) of $\chi^{2} /$ dof $=24 / 22$ and $\chi^{2} /$ dof $=$ $51 / 51$ during Observations 1 and 2, respectively. The lack of variability during both observations allows for time-averaged spectra to be accumulated for analysis.

As the Comptonization modeling requires an accurate spectral shape at high energies, all NUSTAR spectra were re-binned to have a minimum signal-to-noise ratio of five in each spectral bin. For Observation 1, this limits the energy range of the spectra to 3-65 keV (FPMA) and 3-63 keV (FPMB). The data from the longer Observation 2 is limited to the energy range of $3-68 \mathrm{keV}$ (FPMA) and 3-65 keV (FPMB).

3C 382 is one of the targets of NuSTAR's serendipitous survey program (Alexander et al. 2013; Harrison et al. 2013; Baloković et al. 2014) that is utilizing bright Swift-BAT sources as a means to perform a shallow, wide-field survey. As part of this program, Swift-XRT performed a $6.6 \mathrm{ks}$ simultaneous observation of 3C 382 during Observation 1. The Swift-XRT data were reduced and a time-averaged spectrum extracted by automatic analysis tools produced by the Swift-XRT team (Evans et al. 2009). The response matrix used for the analysis was swxpc0to12s6_20010101v013.rmf. To facilitate $\chi^{2}$ fitting, the Swift-XRT spectrum was grouped to a minimum of 25 counts/bin, and, due to a lack of counts at high energies, only data between 0.3 and $7 \mathrm{keV}$ are included in the analysis.

The Swift-UV/Optical Telescope (UVOT) was also operating during the observation and cycled through the V, B, U, UW1, UM2, and UW2 filters, taking one image for each of the filters. The UVOT data were downloaded from the HEASARC 



Figure 2. Left: the top panel plots the count rate spectrum of 3C 382 and the background obtained from Observation 1 for the NuSTAR FPMA (black) and FPMB (red) detectors. The Swift-XRT data are shown in green. The lower panel shows the residuals (in units of standard deviations) when the spectra are fit with a power law modified by Galactic absorption and a weak warm absorber. The data between 4 and $7.5 \mathrm{keV}$ were not included in the fit. Right: similar to the left-hand panel, but for Observation 2

(A color version of this figure is available in the online journal.)

website and the uvotsource tool was used to perform aperture photometry. The tool returns the coincidence loss-corrected fluxes for a given source and background region. Here, the source region was selected to be circular with a 5.0 arcsec radius and centered on 3C 382. The background flux was obtained from a source-free circular region with a 20 arcsec radius in the vicinity of the source. The optical/UV fluxes were corrected for Galactic extinction using the reddening laws of Cardelli et al. (1989) and O'Donnell (1994) with a $E(B-V)=0.0598$ (Schlegel et al. 1998). Assuming $R_{V}=$ 3.16 and the same reddening law, the fluxes were then corrected for the internal extinction of $E(B-V) \approx 0.23$ (Tadhunter et al. 1986). The resulting fluxes are used to analyze the multiwavelength spectral energy distribution (SED) of 3C 382 during Observation 1 (Section 4.4). There is likely a small contribution from the host galaxy in the derived fluxes, however it is clear that the host galaxy of $3 \mathrm{C} 382$ is weak at UV wavelengths and the flux is dominated by the nucleus (Allen et al. 2002), thus we omit any host correction for the purpose of the simple comparison with the predicted SEDs.

XSPEC v.12.8.11 (Arnaud 1996) is used for all X-ray spectral fitting. Spectra from FPMA and FPMB are fit simultaneously for both NUSTAR observations, with a normalization constant left free to account for the slight calibration differences between the two modules. The Swift-XRT spectrum is included in the analysis of Observation 1, with an additional normalization constant left free to vary in the fits. $\chi^{2}$ statistics are used to determine the best model description of the data, and a $\Delta \chi^{2}=2.71$ criterion (i.e., a $90 \%$ confidence range for one parameter of interest) is used to determine the error-bars. The following cosmological parameters are assumed: $\Omega_{M}=0.27$, $\Omega_{\Lambda}=0.73$, and $H_{0}=70 \mathrm{~km} \mathrm{~s}^{-1} \mathrm{Mpc}^{-1}$.

\section{SPECTRAL ANALYSIS}

Galactic absorption with a column density of $N_{\mathrm{H}}=6.98 \times$ $10^{20} \mathrm{~cm}^{-2}$ (Kalberla et al. 2005) is included in all spectral models using the TBabs model (Wilms et al. 2000). In addition, 3C 382 is observed to have a weak, highly ionized warm absorber with $N_{\mathrm{H}} \approx 1.4 \times 10^{21} \mathrm{~cm}^{-2}$ and $\log \xi=2.5$, where $\xi$ is the ionization parameter of the absorbing gas (Torresi et al.

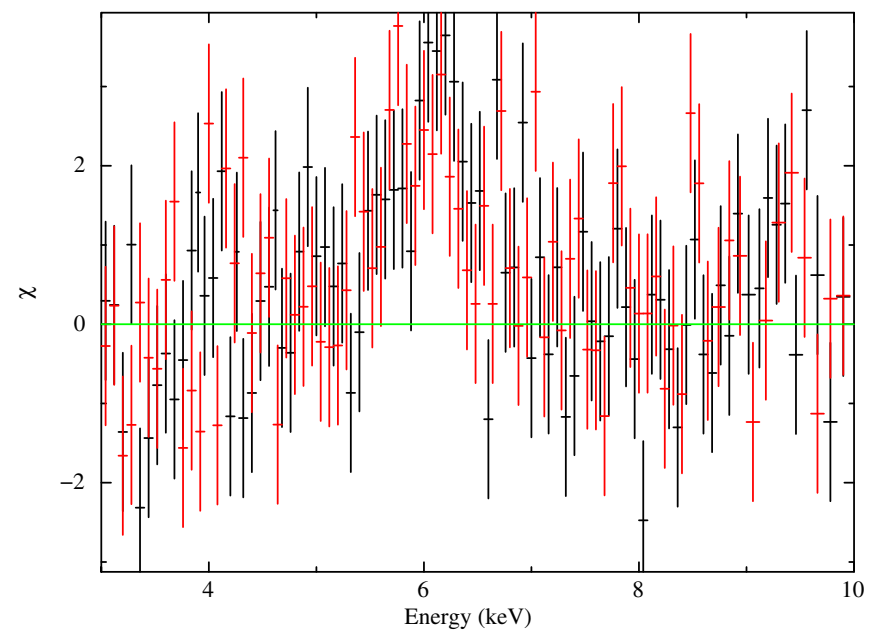

Figure 3. Same as in Figure 2 (right), but zooming in and rebinning to emphasize the residuals in the $\mathrm{Fe} \mathrm{K} \alpha$ band.

(A color version of this figure is available in the online journal.)

2010; Sambruna et al. 2011; Walton et al. 2013). Although the effects of the warm absorber are minor in the NUSTAR band, it is included in the spectral model (with the above parameters) using a grid of XSTAR models calculated by Walton et al. (2013). High resolution Chandra observations of 3C 382 indicate a diffuse halo of emission peaking at a radius of $\approx 10^{\prime \prime}-20^{\prime \prime}$ from the point source (Gliozzi et al. 2007). The temperature and luminosity of this emission is too low to be important in the NuSTAR energy band, nor can it account for the observed soft excess (Torresi et al. 2010), so it is omitted from the spectral modeling.

\subsection{Observation 2-Low Flux}

We begin by analyzing the NUSTAR spectra from Observation 2 as it has the largest number of counts. The right-hand panel of Figure 2 presents the count spectra and the residuals to a simple power-law fit when the 4-7.5 keV data are ignored. The fit is poor $\left(\chi^{2} /\right.$ dof $\left.=1126 / 991\right)$, with a clear residual at the energy of the $\mathrm{Fe} \mathrm{K} \alpha$ line (Figure 3) and signs of a turn down at high energies. Replacing the power law with a cutoff 
Table 2

3C 382 Spectral Fitting Results

\begin{tabular}{|c|c|c|}
\hline & Observation 1 & Observation 2 \\
\hline \multicolumn{3}{|c|}{ Cutoff power law $+6.4 \mathrm{keV}$ Gaussian } \\
\hline $\bar{\Gamma}$ & $1.78_{-0.03}^{+0.02}$ & $1.68_{-0.02}^{+0.03}$ \\
\hline$E_{\text {cut }}(\mathrm{keV})$ & $>190$ & $214_{-63}^{+147}$ \\
\hline$\sigma(\mathrm{keV})$ & $0.37_{-0.2}^{+0.3}$ & $0.34_{-0.12}^{+0.15}$ \\
\hline Fe K $\alpha$ flux (photons $\mathrm{cm}^{-2} \mathrm{~s}^{-1}$ ) & $4.8_{-2.4}^{+2.5} \times 10^{-5}$ & $4.1_{-0.9}^{+1.1} \times 10^{-5}$ \\
\hline $\mathrm{EW}(\mathrm{eV})$ & $79_{-44}^{+52}$ & $114_{-26}^{+30}$ \\
\hline$\chi^{2} /$ dof & $780 / 786$ & $980 / 988$ \\
\hline \multicolumn{3}{|c|}{ compps + 6.4 keV Gaussian } \\
\hline$k T_{e}(\mathrm{keV})$ & $231_{-88}^{+50}$ & $330 \pm 30$ \\
\hline$y$ & $0.40_{-0.06}^{+0.07}$ & $0.38_{-0.05}^{+0.04}$ \\
\hline$\tau$ & 0.23 & 0.15 \\
\hline$\sigma(\mathrm{keV})$ & $0.39_{-0.25}^{+0.27}$ & $0.29_{-0.11}^{+0.14}$ \\
\hline Fe K $\alpha$ flux (photons $\mathrm{cm}^{-2} \mathrm{~s}^{-1}$ ) & $4.5_{-2.4}^{+2.6} \times 10^{-5}$ & $3.5_{-0.8}^{+1.0} \times 10^{-5}$ \\
\hline $\mathrm{EW}(\mathrm{eV})$ & $75_{-52}^{+55}$ & $98 \pm 25$ \\
\hline$\chi^{2} /$ dof & $778 / 786$ & $976 / 988$ \\
\hline$F_{2-10 \mathrm{keV}}\left(\mathrm{erg} \mathrm{cm}^{-2} \mathrm{~s}^{-1}\right)$ & $5 \times 10^{-11}$ & $2.9 \times 10^{-11}$ \\
\hline
\end{tabular}

Notes. A Galactic absorber with $N_{\mathrm{H}}=6.98 \times 10^{20} \mathrm{~cm}^{-2}$ (Kalberla et al. 2005) is included in all fits using the TBabs model (Wilms et al. 2000). Similarly, a weak warm absorber with $N_{\mathrm{H}}=1.4 \times 10^{21} \mathrm{~cm}^{-2}$ and $\log \xi=2.5$ (e.g., Torresi et al. 2010) is also included in all fits using a grid of XSTAR models (Walton et al. 2013). Fits to Observation 1 included a blackbody component to account for the soft excess below $\approx 0.6 \mathrm{keV}$. The blackbody temperature and normalization are consistent with those found by Sambruna et al. (2011): $k T \approx 0.1 \mathrm{keV}$ and normalization $\approx 10^{-4}$. The $\mathrm{Fe} \mathrm{K} \alpha$ line energy is fixed at $6.4 \mathrm{keV}$ in these models. The seed photon temperature for the compps models is fixed at $8.9 \mathrm{eV}$, and a diskbb spectrum is assumed. $\tau$ is estimated using the best fit $k T_{e}$ and $y$.

power law improves the fit to $\chi^{2} / \mathrm{dof}=1108 / 990$ (significant at $>99.99 \%$ according to the $F$-test) with $E_{\text {cut }}=202_{-58}^{+132} \mathrm{keV}$. The addition of a narrow Gaussian $\mathrm{Fe} \mathrm{K} \alpha$ line, fixing its width to $\sigma=90 \mathrm{eV}$ (Gliozzi et al. 2007) and energy to $6.4 \mathrm{keV}$, further improves the fit $\left(\chi^{2} /\right.$ dof $=995 / 989$; significant at $\gg 99.99 \%$ according to the $F$-test). While allowing the energy of the line to float does not significantly improve the fit, thawing the width of the line results in dropping the $\chi^{2}$ to $\chi^{2} /$ dof $=980 / 988$ with $\sigma=0.34_{-0.12}^{+0.15} \mathrm{keV}$ ( $F$-test probability $\left.>99.9 \%\right)$. The cutoff energy increases to $E_{\text {cut }}=214_{-63}^{+147} \mathrm{keV}$. At this stage, no significant residuals are observed in the data. Re-introducing the narrow $(\sigma=90 \mathrm{eV}) \mathrm{Fe} \mathrm{K} \alpha$ line to the model with the broadened line does not result in any improvement to the fit. The best fit parameters from the simple cutoff power law plus Gaussian model are shown in Table 2, and contours of line-flux versus line energy are shown in Figure 4.

To attempt to further characterize the $\mathrm{Fe} \mathrm{K} \alpha$ line and any associated reflection continuum, the cutoff power law is replaced with a pexrav model (Magdziarz \& Zdziarski 1995) where the abundances are fixed at solar and assuming an inclination angle of $40 \mathrm{deg}$ (Giovannini et al. 2001). However, no improvement is found with only an upper-limit to the reflection fraction of $R<0.03$ (the best-fit value of $R$ is $10^{-15}$ ). Replacing pexrav with other reflection models that include the $\mathrm{Fe} \mathrm{K} \alpha$ line, such as pexmon (Nandra et al. 2007) or relxill (García et al. 2014), and continuing to assume solar abundances, result in significantly worse values of $\chi^{2}$ and $R \sim 0.1-0.2$. Allowing the iron abundance to fit freely improves the fit $\left(\chi^{2} / \mathrm{dof}=983 / 986\right.$

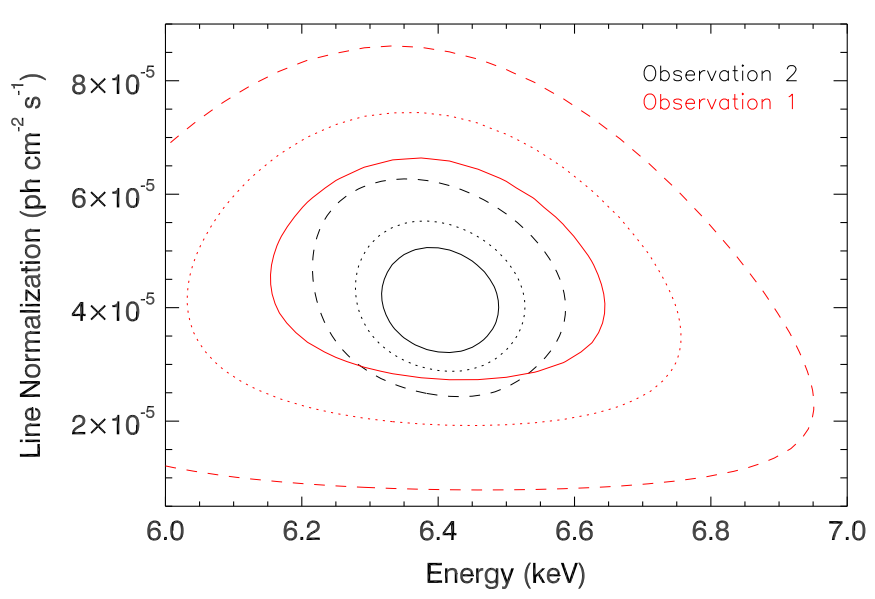

Figure 4. 68\% (solid), 90\% (dotted), and 95\% (dashed) joint confidence contours on the $\mathrm{Fe} \mathrm{K} \alpha$ line flux and energy calculated for Observations 1 (red) and 2 (black) using the cutoff power law plus Gaussian line model. Both observations measure a similar line flux and energy. The line normalization is also consistent with many earlier measurements from 3C 382 (Section 4.1).

(A color version of this figure is available in the online journal.)

for relxill), but the iron abundance is constrained to be $>8.6 \times$ the Solar value, and the reflection fraction remains very small ( $R=0.07 \pm 0.02)$. The best fit ionization parameter from the relxill model is $\log \xi=0.31_{-0.31}^{+1.41}$. This produces a neutral reflection spectrum, consistent with the line energy of $\approx 6.4 \mathrm{keV}$ found by the Gaussian fits.

Relativistic effects can broaden the $\mathrm{Fe} \mathrm{K} \alpha$ line if it originates from the inner accretion disk (e.g., Fabian et al. 1989; Laor 1991; Reynolds \& Nowak 2003). In this situation, the width of the line can be translated into a minimum radius of the reflecting region along the accretion disk. To check if relativistic effects are causing the observed line width in 3C 382 the Gaussian $\mathrm{Fe} \mathrm{K} \alpha$ line in the cutoff power-law model was replaced with a relativistic line profile (using relline; Dauser et al. 2010). The relline model produces a good fit $\left(\chi^{2} / \mathrm{dof}=981 / 988\right)$ and gives an inner radius of $82_{-36}^{+94} r_{g}$ (where $r_{g}=G M / c^{2}$ is the gravitational radius of a black hole with mass $M$ ) for an inclination angle of $40 \mathrm{deg}$ and an unbroken emissivity index of -3 . This result indicates that the detected line likely does not originate from the inner accretion disk. Letting the inclination angle or emissivity profile vary does not improve the fit and yields only lower limits to the parameters (inclination angle $>25$ deg; emissivity index $>2.25$ ). No evidence for a weak relativistic line from closer to the inner disk is found in the data. The relxill model mentioned above also includes relativistic blurring and a similar constraint on the inner radius $\left(>57 r_{g}\right)$ was found by that model.

The very weak reflection continuum found in this observation of 3C 382 presents an opportunity to determine if the continuum from a broad-line radio galaxy is described by thermal Comptonization models similarly to radio-quiet Seyfert galaxies. We therefore remove the cutoff power-law component and replace it with a compps mode ${ }^{13}$ (Poutanen \& Svensson 1996), assuming no reflection component. The seed photons are assumed to arise from a multi-temperature blackbody (the diskbb model) with a maximum temperature of $8.9 \mathrm{eV}$, the characteristic temperature of an accretion disk around a $10^{9} M_{\odot}$ black hole (Marchesini et al. 2004), assuming accretion at $20 \%$ of the Eddington limit 13 Models using comptt (Titarchuk 1994) provide similar fits, but with much
larger uncertainties on the parameters. 
(Gliozzi et al. 2007). In addition to the plasma temperature $k T_{e}$, the Compton $y$ parameter, defined as $y=4 \tau\left(k T_{e} / 511 \mathrm{keV}\right)$, is used as a fit parameter in place of the optical depth $\tau$. The compps model predicts spectra for several different geometries of the Comptonizing plasma (e.g., slab, sphere, or cylinder). None of the geometries give qualitatively different results, so we focus here on the fits from the slab geometry with seed photons injected at the bottom of the slab, as it gives the best constraints on the parameters, and it is one of the two geometries that is accurately computed by compps (rather than approximated; Poutanen \& Svensson 1996). The spectral model with compps and an $\mathrm{Fe} \mathrm{K} \alpha$ line provides an excellent fit to the data $\left(\chi^{2} /\right.$ dof $\left.=976 / 988\right)$ and gives the temperature of the Comptonizing electrons to be $k T_{e}=330 \pm 30 \mathrm{keV}$ and a Compton $y$ parameter of $0.38_{-0.05}^{+0.04}$ (see Table 2). Together, these values result in an optical depth of $\tau=0.15$. The $2-10 \mathrm{keV}$ flux of $3 \mathrm{C} 382$ determined by this fit is $F_{2-10 \mathrm{keV}}=2.9 \times 10^{-11} \mathrm{erg}$ $\mathrm{cm}^{-2} \mathrm{~s}^{-1}$, which corresponds to an unabsorbed luminosity of $L_{2-10 \mathrm{keV}}=2.3 \times 10^{44} \mathrm{erg} \mathrm{s}^{-1}$.

\subsection{Observation 1-High Flux}

The analysis of the short Observation 1 is significantly improved with the inclusion of the Swift-XRT data. The left-hand panel of Figure 2 plots the Swift and NuSTAR count rate spectra, as well as the residuals to a power-law fit (ignoring the $4-7.5 \mathrm{keV}$ band $)$. The fit is adequate $\left(\chi^{2} / \mathrm{dof}=810 / 791\right)$, but there are clear positive residuals at $\lesssim 0.7 \mathrm{keV}$, and hints of an $\mathrm{Fe} \mathrm{K} \alpha$ line. The soft excess has been seen in earlier observations of 3C 382 (e.g., Sambruna et al. 2011), and is well modeled by a blackbody component. The addition of the blackbody significantly improves the fit (new $\chi^{2} /$ dof $=796 / 789$; $F$-test probability $>99.9 \%$ ) and gives a temperature of $k T=$ $0.09_{-0.02}^{+0.03} \mathrm{keV}$ and normalization ${ }^{14} \approx 1.3 \times 10^{-4}$, consistent with previous measurements (Sambruna et al. 2011). These values change very little for the different models of the high energy emission. The addition of a high-energy cutoff makes only a marginal improvement to the fit $\left(\chi^{2} /\right.$ dof $=793 / 788 ; F$-test probability $\approx 90 \%)$ with $E_{\text {cut }}>167 \mathrm{keV}$. A narrow $(\sigma=90 \mathrm{eV})$ $\mathrm{Fe} \mathrm{K} \alpha$ line at $6.4 \mathrm{keV}$ results in $\chi^{2} / \mathrm{dof}=784 / 787$ which is significant at the $99.7 \%$ level according to the $F$-test. Allowing $\sigma$ to vary reduces $\chi^{2}$ to 780 for 786 dof (96\% significant) and gives $\sigma=0.37_{-0.2}^{+0.3} \mathrm{keV}$. The lower-limit to the cutoff energy now rises to $190 \mathrm{keV}$. The flux of the line is $\approx 30 \%$ larger than in Observation 2 (see Table 2), but is consistent with a constant flux across both observations (Figure 4). Although the high-energy cutoff is not strictly necessary for this fit, we show the parameters of this model in the upper-half of Table 2 to compare with the results from Observation 2. As with Observation 2, pexrav only gives an upper limit to the reflection fraction $R<0.16$ (the best fit value of $R$ is 0.03 , but is consistent with 0 within the errors). The pexmon and relxill models both give good fits $\left(\chi^{2} /\right.$ dof $=787 / 787$ and $786 / 785$, respectively $)$ with solar abundances, but with low reflection fractions $(0.13 \pm 0.09$ for the pexmon model and $0.10_{-0.06}^{+0.07}$ for the relxill model). The relxill model yields an upper limit to the ionization parameter of $\log \xi<3.3$, and is unable to constrain an inner radius from disk reflection. Given the poor statistics of this short observation, no further modeling of the $\mathrm{Fe} \mathrm{K} \alpha$ line was attempted.

The combination of NUSTAR and Swift-XRT produces a spectrum that spans over two orders of magnitude in energy

\footnotetext{
14 Equal to $L_{39} / D_{10}^{2}$ where $L_{39}$ is the luminosity of the blackbody in units of $10^{39} \mathrm{erg} \mathrm{s}^{-1}$, and $D_{10}$ is the distance to $3 \mathrm{C} 382 \mathrm{in}$ units of $10 \mathrm{kpc}$.
}

which, in combination with the weak reflection continuum, provides a significant lever arm for potentially constraining thermal Comptonization models (even with the weak constraint on $\left.E_{\text {cut }}\right)$. Applying the same compps model as in Observation 2 yields an excellent fit to Observation $1\left(\chi^{2} /\right.$ dof $\left.=778 / 786\right)$ with $k T_{e}=231_{-88}^{+50} \mathrm{keV}$ and $y=0.40_{-0.06}^{+0.07}$, corresponding to $\tau=0.23$ (Table 2). The $2-10 \mathrm{keV}$ flux and unabsorbed luminosity obtained by this model is $5 \times 10^{-11} \mathrm{erg} \mathrm{cm}^{-2} \mathrm{~s}^{-1}$ and $4 \times 10^{44} \mathrm{erg} \mathrm{s}^{-1}$, respectively. Thus, 3C 382 was $1.7 \times$ fainter in Observation 2 than in Observation 1.

\section{DISCUSSION AND CONCLUSIONS}

\subsection{Comparison to Previous Results}

The results from the $N U S T A R$ observations agree with many of the previous measurements of 3C 382 (e.g., Grandi et al. 2001). In particular, the long Suzaku observation analyzed by Sambruna et al. (2011) and Walton et al. (2013) is the highest quality spectrum taken of $3 \mathrm{C} 382$ and these authors find a similarly small value of the reflection fraction $(R \approx 0.1-0.15)$. Sambruna et al. (2011) combine the Suzaku data with the integrated Swift-BAT spectrum to measure a cutoff energy of $E_{\text {cut }}=175_{-20}^{+25} \mathrm{keV}$, consistent with both NuSTAR measurements (Table 2). The photon index and flux obtained from the Suzaku data were $\Gamma=1.74$ and $F_{2-10 \mathrm{keV}}=4.1 \times 10^{-11} \mathrm{erg} \mathrm{cm}^{-2} \mathrm{~s}^{-1}$ (Sambruna et al. 2011). These values nicely fall in the middle of the two NUSTAR observations and support the assertion that 3C 382 follows the typical Seyfert pattern of softening as it brightens.

The Fe K $\alpha$ line flux is measured to be $\approx 4 \times 10^{-5} \mathrm{ph} \mathrm{cm}^{-2} \mathrm{~s}^{-1}$ in both observations despite a change in continuum flux by nearly a factor of two. Previous observations with instruments with similar energy resolutions at $6.4 \mathrm{keV}$ all find line fluxes at approximately this value (Eracleous et al. 2000; Grandi et al. 2001; Gliozzi et al. 2007). Indeed, Grandi et al. (2001) noted that line flux has remained constant in all earlier observations despite large changes in the continuum flux, and argued that the line must result from a distant reprocessor. The NuSTAR observations are consistent with this interpretation; however, this conclusion does not exclude the presence of a weak, relativistically broadened component to the line as described by Sambruna et al. (2011). To test this possibility, we attempted to model Observation 2 with one broad and one narrow Gaussian to model the $\mathrm{Fe} \mathrm{K} \alpha$ line complex. While a good fit is achieved, the $\chi^{2}$ is unchanged from the single Gaussian model, and the normalization of the narrow $6.4 \mathrm{keV}$ line is consistent with zero. A similar result is achieved when attempting a double reflection model using two relxill models; namely, the normalization of the second reflector was sent towards zero. Again, this is not evidence that this model is incorrect, but our data around the $\mathrm{Fe}$ $\mathrm{K} \alpha$ line does not allow us to test these complex models.

\subsection{A Comptonizing Corona}

The spectral analysis of the NUSTAR data presents evidence that the X-ray spectrum of the BLRG 3C 382 exhibits a Seyfertlike Comptonizing corona; specifically, the corona cools and produces a softer spectrum when it brightens (Table 2). This behavior is inferred to be typical for non-jetted Seyfert 1 galaxies (e.g., Lubiński et al. 2010; Veledina et al. 2011), but is not observed in AGNs with jet-dominated continua (e.g., 3C 273; Chernyakova et al. 2007). ${ }^{15}$ The X-ray spectra of jet-dominated

\footnotetext{
15 Interestingly, some X-ray binaries also do not show the

"softer-when-brighter" behavior when in the jet-dominated state (e.g., Gandhi et al. 2008).
} 


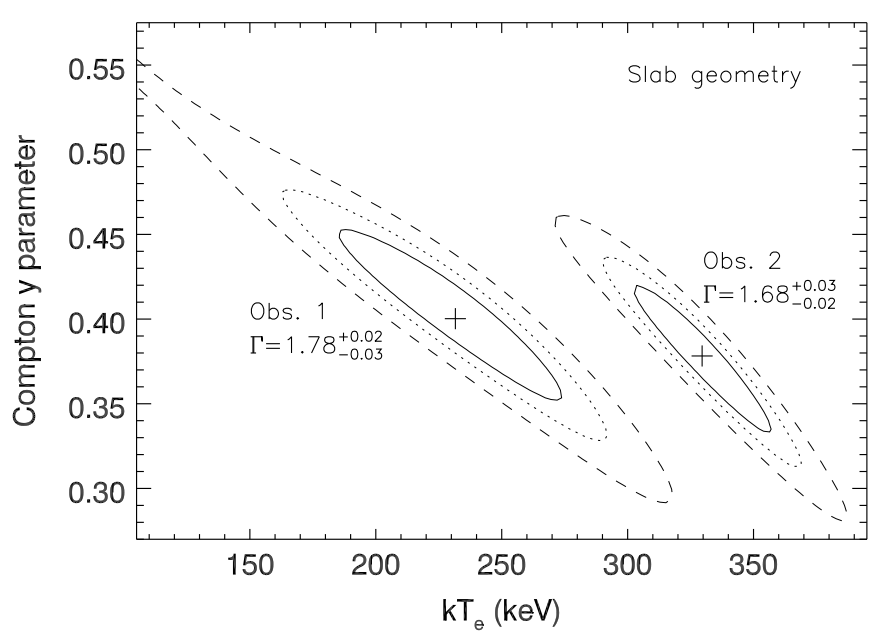

Figure 5. 68\% (solid), 90\% (dotted), and 95\% (dashed) joint confidence contours on $k T_{e}$ and $y$ calculated for Observations 1 and 2 from the best fitting compps $+6.4 \mathrm{keV}$ Gaussian models (Table 2). The crosses mark the location of the best fit values.

AGNs (e.g., blazars) typically have $\Gamma<1.5$ (e.g., Sambruna et al. 2006; Gianní et al. 2011), produce hard X-ray variations $\approx 2 \times$ larger than Seyferts (e.g., Chernyakova et al. 2007; Soldi et al. 2014), and are gamma-ray sources rather than exhibiting cutoffs at $\sim 100-200 \mathrm{keV}$ (Giommi et al. 2006). In contrast, 3C 382 is not a Fermi source (Nolan et al. 2012), has hard X-ray variability properties similar to Seyfert 1s (Soldi et al. 2014), and has a detectable X-ray spectral cutoff. The hard X-ray properties of $3 \mathrm{C} 382$ revealed by $N U S T A R$ are therefore entirely consistent with those found from non-jetted Seyfert $1 \mathrm{~s}$.

To examine the changes inferred in the corona properties of 3C 382 more closely, confidence contours for $k T_{e}$ and $y$ are computed using the best fitting compps model and are overplotted in Figure 5. The contours show that the change in coronal temperature between the two observations is $\approx 90 \%$ significant, and that the corona is cooler in the more luminous state (e.g., Haardt et al. 1997; Grandi et al. 2001). This change in temperature is consistent with the model of a thermal Comptonizing corona producing the X-ray spectrum in 3C 382. Interestingly, the value of $k T_{e}$ derived from the compps model of Observation 2 is larger than the common approximations $k T_{e} \approx E_{\text {cut }} / 2$ (for $\tau \lesssim 1$ ) or $k T_{e} \approx E_{\text {cut }} / 3$ (for $\tau \gg$ 1). ${ }^{16}$ This mismatch has been seen before by Petrucci et al. (2001) and is simply explained by recalling that cutoff powerlaws are only approximations to the spectra produced by thermal Comptonization spectra. Geometric effects due to the anisotropic nature of the Comptonization process will introduce a slight curvature to the spectrum at high energies (Stern et al. 1995; Poutanen \& Svensson 1996). The effect is especially important for corona with high $k T_{e}$ and low $\tau$ (Petrucci et al. 2000, 2001). Interestingly, the compps fits yield lower $\chi^{2}$ than the cutoff power-law model for both 3C 382 data sets indicating that this curvature may indeed be in the data. Moreover, although $k T_{e}$ and $\tau$ remain similar, compps models where the seed photons are distributed isotropically in the hot plasma result in $\Delta \chi^{2}=+5$ compared to the anisotropic model reported in Table 2 . This result provides tantalizing evidence

\footnotetext{
16 Observation 2 can be well fit with a low- $k T_{e}$, high- $\tau$ model (e.g., $k T_{e} \approx 41 \mathrm{keV}$ and $\tau \approx 2.5$ assuming spherical geometry), but with a larger $\chi^{2}$ than the high- $k T_{e}$ fit discussed here $\left(\Delta \chi^{2} \approx+11\right)$. We therefore focus on the latter model in this paper.
}

that anisotropic Comptonization is playing an important role in 3C 382, and strongly supports the use of sophisticated Comptonization models such as compps when interpreting the high-energy cutoffs of AGNs. In particular, Comptonization modeling of AGNs with low $R$ may prove especially useful in determining details of the corona.

\subsection{Comparison to Other Coronal Measurements}

Currently, NUSTAR has measured the coronal parameters in two other AGNs: the Seyfert 1 galaxy IC 4329A $\left(k T_{e}=\right.$ $61 \pm 1 \mathrm{keV}$ and $\tau=0.68 \pm 0.02$; Brenneman et al. 2014b) and the narrow-line Seyfert 1 Swift J2127.4+5654 $\left(k T_{e}=\right.$ $68_{-32}^{+37} \mathrm{keV}$ and $\tau=0.35_{-0.19}^{+0.35}$; Marinucci et al. 2014). Both these measurements assume a slab geometry and were performed using the comptt model. 3C 382 with a $k T_{e} \gtrsim 150 \mathrm{keV}$ appears to have a significantly hotter corona than either of these sources, and, potentially, a more tenuous corona than IC 4329A. However, Matt et al. (2014) also report a high temperature corona $\left(k T_{e} \approx 110-210 \mathrm{keV}\right)$ in joint $X M M$ Newton-NuSTAR fits of the Seyfert 1 Ark 120. Thus, at this early stage, we cannot draw a conclusion about the importance of the hot corona observed in $3 \mathrm{C} 382$, but $\approx 20$ bright AGNs have been targeted by $N u S T A R$ with the goal of determining the coronal parameters (Harrison et al. 2013). This sample will allow interesting comparisons of coronal temperatures and optical depths among AGNs of different classes.

\subsection{Implications on Coronal Geometry and Dynamics}

Recent observational innovations utilizing $\mathrm{Fe} \mathrm{K} \alpha$ reverberation and microlensing have allowed the first constraints to be placed on the size of the X-ray corona in radio-quiet AGNs (e.g., Chartas et al. 2009; Zoghbi et al. 2012; Wilkins \& Fabian 2013). These techniques all point to a small, centrally concentrated corona, situated within $\approx 20 r_{g}$ from the black hole (Reis $\&$ Miller 2013). Such compact coronae provide the necessary illumination of the inner disk to produce a relativistic Fe $\mathrm{K} \alpha$ line (Fabian et al. 2014). Although the 3C 382 spectra analyzed here do not show strong evidence for a relativistic line, we can combine the Comptonization modeling and the simultaneous Swift data during Observation 1 to obtain a simple view of the $\mathrm{X}$-ray corona of 3C 382. Figure 6 shows the optical/UV/X-ray SED of 3 C 382 predicted by the best fitting compps models of Observation 1. The different lines show how the predicted SED varies for different coronal geometries. All of these models assume the same seed photon spectrum (a diskbb with maximum $k T=8.9 \mathrm{eV}$ ) and give the same $\chi^{2}$ to the X-ray data. The data points in the figure are the de-reddened fluxes of 3C 382 obtained from the simultaneous Swift observation (see Section 2). A least-squares fit to the Swift-UVOT data results in $f_{v} \propto v^{0.2 \pm 0.1}$ (dotted line in Figure 6), consistent with the $f_{v} \propto v^{0.3}$ slope expected for a Shakura-Sunyaev accretion disk (e.g., Frank et al. 2002). Interestingly, the amplitude of the SwiftUVOT data is $\sim 10 \times$ larger than the compps models. The UV emission predicted by the Comptonization models is from the zeroth-scattering order, which, in a low $\tau$ corona such as seen in $3 \mathrm{C} 382$, is nearly equal to the total UV emission necessary to produce the observed X-rays. Thus, the SED models indicate that the X-ray corona may intercept only $\sim 10 \%$ of the accretion disk UV flux, broadly consistent with models of patchy corona in Seyfert galaxies (e.g., Haardt et al. 1994).

Evidence for a Comptonizing corona in 3C 382, when combined with the fact that the high-energy variability properties 


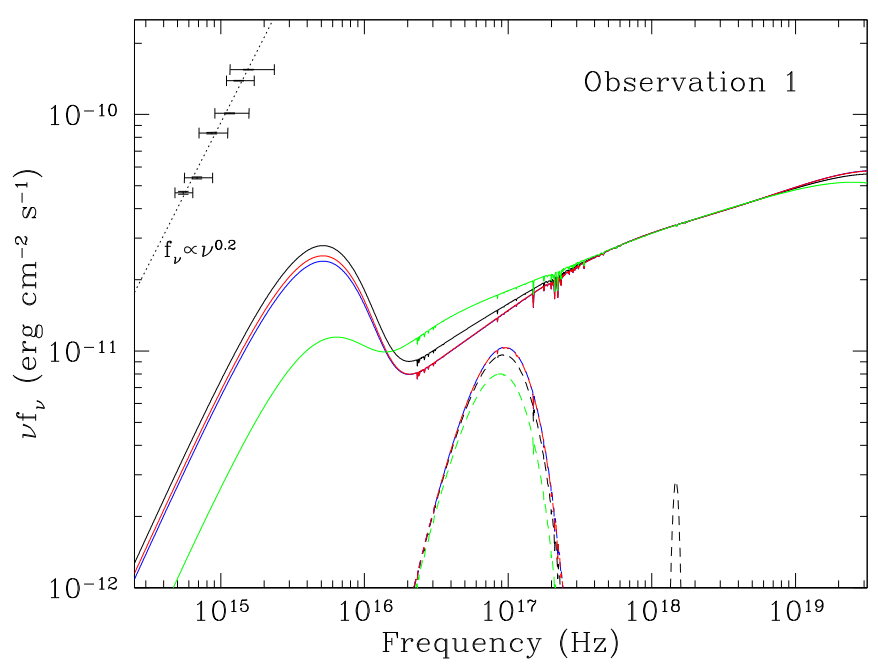

Figure 6. Predicted optical/UV/X-ray SED for Observation 1 of 3C 382 as determined by the compps modeling of the X-ray data. Galactic absorption has been removed from the models, but the small effects of the warm absorber are still visible. To highlight the Comptonization spectra, the full SED has been decomposed into the compps continuum (solid lines), the soft excess and the Fe $\mathrm{K} \alpha$ line (dashed lines). The various lines show how the predictions depend on different coronal geometries: slab (black), cylinder (blue), hemisphere (red), and sphere (green). The same seed photon distribution (diskbb with $k T=8.9 \mathrm{eV}$ ) was used for all the Comptonization models. The data points are from the Swift UVOT observations during Observation 1, and have been corrected for Galactic and internal extinction (see Section 2).

(A color version of this figure is available in the online journal.)

of BLRGs are consistent with those from non-jetted Seyfert 1s (Soldi et al. 2014), shows that contamination from jets in the X-ray band of BLRGs has likely been minimal. Thus, there must be another explanation for the extremely weak reflection features of $3 \mathrm{C} 382$. Interestingly, the source does present a significant $(\mathrm{EW} \approx 100 \mathrm{eV}) \mathrm{Fe} \mathrm{K} \alpha$ line, but it does not originate from the inner accretion disk, nor does it arise from the broad line region (the Fe K $\alpha$ FWHM $\sim 30,000 \mathrm{~km} \mathrm{~s}^{-1}$, compared to $\approx 12,000 \mathrm{~km} \mathrm{~s}^{-1}$ for the $\mathrm{H} \alpha$ line; Eracleous \& Halpern 1994). However, the line width is only slightly larger than the energy resolution (400 eV FWHM; Harrison et al. 2013), so it is possible that the observed $\mathrm{Fe} \mathrm{K} \alpha$ line width and strength is caused by the blending of multiple line components. Regardless of the number of components to the line, there does not seem to be a significant reflection continuum in the observed spectrum. This fact points to an origin of the line in material with a large $\mathrm{Fe}$ abundance or from Compton thin clouds that may originate in a dusty outflow from the nucleus (e.g., Hönig et al. 2013).

If the X-ray source in 3C 382 is a Comptonizing corona similar to Seyfert galaxies, then the lack of reflection from the inner disk is still puzzling. The inner accretion disk must be either blocked from view, absent, or emit such a weak reflection continuum that it cannot be identified in our data. Sambruna et al. (2011) present evidence for a highly ionized inner accretion disk in 3 C 382 that, when combined with a distant reflector that fits the narrow component of the $\mathrm{Fe} \mathrm{K} \alpha$ line, also accounts for the soft excess. Unfortunately, our data quality is not high enough to directly test this model, although we find a slightly broader neutral $\mathrm{Fe} \mathrm{K} \alpha$ line than Sambruna et al. (2011) in both observations. Another possibility for the extremely weak disk reflection is a transition to an optically thin, radiatively inefficient accretion flow inside $\sim 70-100 r_{g}$ as a result of a 'jet cycle' where the inner disk is 'emptied' during a jet outburst (e.g., Lohfink et al. 2013). However, there was no radio monitoring of $3 \mathrm{C} 382$ during the NUSTAR observations, so this model cannot be tested for this source. Finally, if the $\mathrm{X}$-ray emitting corona is outflowing away from the accretion disk (Beloborodov 1999; Malzac et al. 2001) then it would naturally explain the hard power-law slope and the apparently very weak disk reflection (see also Fabian et al. 2014). The models of Malzac et al. (2001) indicate that an outflow speed of $\gtrsim 0.5 c$ would yield $\Gamma \sim 1.7$ and $R \lesssim 0.2$. Given that the black hole environment of 3C 382 is producing a relativistic jet, this process, combined with ionized reflection from close to the black hole, may be a promising model to explain the weak reflection features seen in BLRGs. Interestingly, IC 4329A also has weak reflection features and a hard continuum similar to 3C 382 (Brenneman et al. 2014b), but does not produce a large scale radio-jet. Brenneman et al. (2014b) estimate an outflow speed of $\sim 0.2 c$ for that source. These results may be hinting at a deeper connection between the corona and the base of a jet (e.g., Markoff et al. 2005). Further discrimination of the models will require high resolution and broadband X-ray data (with, e.g., $A S T R O-H$ ) combined with contemporaneous radio imaging.

We thank the referee for a helpful report that improved the paper. This work was supported under NASA Contract No. NNG08FD60C, and made use of data from the NuSTAR mission, a project led by the California Institute of Technology, managed by the Jet Propulsion Laboratory, and funded by the National Aeronautics and Space Administration. We thank the NUSTAR Operations, Software and Calibration teams for support with the execution and analysis of these observations. This research has made use of the NuSTAR Data Analysis Software (NuSTARDAS) jointly developed by the ASI Science Data Center (ASDC, Italy) and the California Institute of Technology (USA). This work made use of data supplied by the UK Swift Science Data Centre at the University of Leicester. This research has made use of data, software and/or web tools obtained from NASA's High Energy Astrophysics Science Archive Research Center (HEASARC), a service of Goddard Space Flight Center and the Smithsonian Astrophysical Observatory. D.R.B. acknowledges support from NASA ADAP grant NNX13AI47G and NSF award AST 1008067. A.M. acknowledge financial support from Italian Space Agency under grant ASI/INAF I/037/ 12/0-011/13 and from the European Union Seventh Framework Programme (FP7/2007-2013) under grant agreement n.312789. M.B. acknowledges support from the International Fulbright Science and Technology Award.

\section{REFERENCES}

Alexander, D. M., Stern, D., Del Moro, A., et al. 2013, ApJ, 773, 125 Allen, M. G., Sparks, W. B., Koekemoer, A., et al. 2002, ApJS, 139, 411

Arnaud, K. A. 1996, in ASP Conf. Ser. 101, Astronomical Data Analysis Software and Systems V, ed. G. Jacoby \& J. Barnes (San Francisco, CA: ASP), 17

Ballantyne, D. R. 2007, MPLA, 22, 2397

Ballantyne, D. R., Ross, R. R., \& Fabian, A. C. 2002, MNRAS, 332, L45

Baloković, M., Comastri, A., Harrison, F. A., et al. 2014, ApJ, in press (arXiv:1408.5414)

Beloborodov, A. M. 1999, ApJL, 510, L123

Brenneman, L. W., Madejski, G., Fuerst, F., et al. 2014a, ApJ, 781, 83 Brenneman, L. W., Madejski, G., Fuerst, F., et al. 2014b, ApJ, 788, 61

Cardelli, J. A., Clayton, G. C., \& Mathis, J. S. 1989, ApJ, 345, 245

Chartas, G., Kochanek, C. S., Dai, X., Poindexter, S., \& Garmire, G. 2009, ApJ, 693, 174

Chernyakova, M., Neronov, A., Courvoisier, T. J.-L., et al. 2007, A\&A, 465,147

Dauser, T., Wilms, J., Reynolds, C. S., \& Brenneman, L. W. 2010, MNRAS, 409, 1534 
Eracleous, M., \& Halpern, J. P. 1994, ApJS, 90, 1

Eracleous, M., Sambruna, R. M., \& Mushotzky, R. F. 2000, ApJ, 537, 654

Evans, D. A., Reeves, J. N., Hardcastle, M. J., et al. 2010, ApJ, 710, 859

Evans, P. A., Beardmore, A. P., Page, K. L., et al. 2009, MNRAS, 397, 1177

Fabian, A. C., Parker, M. L., Wilkins, D. R., et al. 2014, MNRAS, 439, 2307

Fabian, A. C., Rees, M. J., Stella, L., \& White, N. E. 1989, MNRAS, 238, 729

Frank, J., King, A., \& Raine, D. 2002, Accretion Power in Astrophysics (3rd ed.; Cambridge: Cambridge Univ. Press)

Gandhi, P., Makishima, K., Durant, M., et al. 2008, MNRAS, 390, L29

García, J., Dauser, T., Lohfink, A., et al. 2014, ApJ, 782, 76

Garofalo, D., Evans, D. A., \& Sambruna, R. M. 2010, MNRAS, 406, 975

Gianní, S., De Rosa, A., Bassani, L. P., et al. 2011, MNRAS, 411, 2137

Giommi, P., Colafrancesco, S., Cavazzuti, E., Perri, M., \& Pittori, C. 2006, A\&A, 445,843

Giovannini, G., Cotton, W. D., Feretti, L., Lara, L., \& Venturi, T. 2001, ApJ, 552,508

Gliozzi, M., Sambruna, R. M., Eracleous, M., \& Yaqoob, T. 2007, ApJ, 664, 88

Grandi, P., Maraschi, L., Urry, C. M., \& Matt, G. 2001, ApJ, 556, 35

Grandi, P., Urry, C. M., \& Maraschi, L. 2002, NewAR, 46, 221

Haardt, F., Maraschi, L., \& Ghisellini, G. 1994, ApJL, 432, L95

Haardt, F., Maraschi, L., \& Ghisellini, G. 1997, ApJ, 476, 620

Harrison, F. A., Craig, W. W., Christensen, F. E., et al. 2013, ApJ, 770, 103

Hönig, S. F., Kishimoto, M., Tristram, K. R. W., et al. 2013, ApJ, 771, 87

Kalberla, P. M. W., Burton, W. B., Hartmann, D., et al. 2005, A\&A, 440, 775

Laor, A. 1991, ApJ, 376, 90

Lohfink, A. M., Reynolds, C. S., Jorstad, S. G., et al. 2013, ApJ, 772, 83

Lubiński, P., Zdziarski, A. A., Walter, R., et al. 2010, MNRAS, 408, 1851

Magdziarz, P., \& Zdziarski, A. A. 1995, MNRAS, 273, 837

Malzac, J., Beloborodov, A. M., \& Poutanen, J. 2001, MNRAS, 326, 417

Marchesini, D., Celotti, A., \& Ferrarese, L. 2004, MNRAS, 351, 733

Marinucci, A., Matt, G., Kara, E., et al. 2014, MNRAS, 440, 2347
Markoff, S., Nowak, M. A., \& Wilms, J. 2005, ApJ, 635, 1203

Matt, G., Marinucci, A., Guianzzi, M., et al. 2014, MNRAS, 439, 3016

Nandra, K., O’Neill, P. M., George, I. M., \& Reeves, J. N. 2007, MNRAS, 382, 194

Nolan, P. L., Abdo, A. A., Ackermnann, M., et al. 2012, ApJS, 199, 31

O’Donnell, J. E. 1994, ApJ, 422, 158

Petrucci, P. O., Haardt, F., Maraschi, L., et al. 2000, ApJ, 540, 131

Petrucci, P. O., Haardt, F., Maraschi, L., et al. 2001, ApJ, 556, 716

Poutanen, J., \& Svennson, R. 1996, ApJ, 470, 249

Reis, R. C., \& Miller, J. M. 2013, ApJL, 769, L7

Reynolds, C. S., \& Nowak, M. A. 2003, PhR, 377, 389

Risaliti, G., Harrison, F. A., Madsen, K. K., et al. 2013, Natur, 494, 449

Sambruna, R. M., Eracleous, M., \& Mushotzky, R. F. 1999, ApJ, 526, 60

Sambruna, R. M., Markwardt, C. B., Mushotzky, R. F., et al. 2006, ApJ, 646, 23

Sambruna, R. M., Reeves, J. N., Braito, V., et al. 2009, ApJ, 700, 1473

Sambruna, R., Tombesi, F., Reeves, J. N., et al. 2011, ApJ, 734, 105

Schlegel, D. J., Finkbeiner, D. P., \& Davis, M. 1998, ApJ, 500, 525

Soldi, S., Beckmann, V., Baumgartner, W. H., et al. 2014, A\&A, 563, A57

Stern, B. E., Poutanen, J., Svennson, R., Sikora, M., \& Begelman, M. C. 1995, ApJL, 449, L13

Tadhunter, C. N., Perez, E., \& Fosbury, R. A. E. 1986, MNRAS, 219, 555

Titarchuk, L. 1994, ApJ, 434, 570

Torresi, E., Grandi, M., Longinotti, A. L., et al. 2010, MNRAS, 401, L10

Veledina, A., Vurm, I., \& Poutanen, J. 2011, MNRAS, 414, 3330

Walton, D. J., Nardini, E., Fabian, A. C., Gallo, L. C., \& Reis, R. C. 2013, MNRAS, 428, 2901

Wilkins, D. R., \& Fabian, A. C. 2013, MNRAS, 430, 247

Wilms, J., Allen, A., \& McCray, R. 2000, ApJ, 542, 914

Zdziarski, A. A., \& Grandi, P. 2001, ApJ, 551, 186

Zoghbi, A., Fabian, A. C., Reynolds, C. S., \& Cackett, E. M. 2012, MNRAS, 422,129 